\title{
3D Formulation for Revolute Clearance Joints
}

\author{
F. Marques ${ }^{1}$, F. Isaac ${ }^{2}$, N. Dourado ${ }^{3}$ and P. Flores ${ }^{4}$ \\ ${ }^{1}$ CMEMS - University of Minho, Portugal, e-mail:fmarques@dem.uminho.pt \\ ${ }^{2}$ CMEMS - University of Minho, Portugal, e-mail: efinhoisaac@hotmail.com \\ ${ }^{3}$ CMEMS - University of Minho, Portugal, e-mail: nunodourado@dem.uminho.pt \\ ${ }^{4}$ CMEMS - University of Minho, Portugal, e-mail: pflores@dem.uminho.pt
}

\begin{abstract}
In this work, a general and comprehensive methodology for the modeling and analysis of spatial multibody systems with revolute clearance joints is presented. The proposed formulation considers the journal and the bearing elements as two cylindrical colliding bodies. All the potential contact scenarios and their kinematics are determined from the relative positions of two cylinders and from the kinematics of the multibody systems. It must be highlighted that both radial and axial clearances are modeled under the new proposed approach. The methodology presented here is demonstrated by using a spatial slider-crank mechanism that incorporates a spatial revolute clearance joint. The results show that the system's response is considerably influenced by the existence of a clearance joint.
\end{abstract}

Key words: clearance joints, contact detection, spatial systems, multibody dynamics.

\section{Introduction}

Over the last decades, several works have addressed the problem of modeling clearance joints, either for spherical, revolute, and translational joints [1]. Although the majority of mechanisms presents spatial motions, most of these studies focuses on planar motion, which can hardly be extrapolated to spatial multibody systems [2]. The implementation of spatial formulation typically involves a large number of contact scenarios and a more complex methodology in what concerns to contact detection. Some authors also include the effects of lubrication and bodies' flexibility, just to mention a few [3].

The purpose of this work is to present a new methodology to model multibody systems with dry spatial revolute clearance joints. A revolute joint is constituted by two mechanical components, namely the bearing and the journal. These two elements are considered to be rigid bodies, which collide, because they have different dimensions due to the presence of clearance. This formulation takes into account the existence of both radial and axial clearance, being the latter often neglected in most of the available studies. In this work, it is considered a pseudo penetration during the collision of the two bodies, therefore, the determination of 
the potential contact points highly affects the evaluation of the forces generated during the contact. In order to correctly evaluate the influence of the clearance in the mechanism's motion, an accurate and efficient methodology must be implemented. The remaining of this paper is organized as follows. Section 2 describes the formulation for the geometry description and contact detection. A spatial slider crank mechanism is used in Section 3 as numerical example of application. Finally, in Section 4 the main conclusion are drawn.

\section{Formulation of Revolute Clearance Joints}

The modeling of a revolute joint with clearance involves the correct definition of the geometry of the contacting bodies. A general revolute joint consists in two cylindrical elements with an inner and outer surfaces of potential contact, respectively, the bearing and journal, as represented in Fig. 1. These two elements belong to different bodies and can be completely defined by the radii, the lengths and their location and orientation on each body which are characterized by a point $P$ in the center of the cylinder's axis and a unit vector a with its orientation. The clearance size can be calculated using the dimensions of the journal and bearing as follows

$$
c_{r}=R_{i}-R_{j} \quad c_{a}=\left(L_{i}-L_{j}\right) / 2
$$

where $c_{r}$ and $c_{a}$ denote the radial and axial clearance, respectively, $R$ represents the radius of the element, and $L$ is the length. From now on, the subscripts $i$ and $j$ refer to the bearing and journal, respectively.

By considering two cylindrical elements, each one contains one cylindrical surface (lateral) and two plane surfaces in the extremities. The combination of different contact types results in a total of thirteen contact scenarios [4]: no contact, 3 aligned configurations, and 9 misaligned scenarios. It is worth to mention that some of these configurations might be impractical due to the clearances' size.

In order to promote an easier contact detection, auxiliary points are considered in the center of each extremity of both elements, as shown in Fig. 1. Thus, the two ends are distinguished between $A$ and $B$. The coordinates of these points can be determined by the following expressions

$$
\begin{aligned}
& \mathbf{r}^{A_{k}}=\mathbf{r}^{P_{k}}+\frac{L_{k}}{2} \mathbf{a}_{k}, \quad(k=i, j) \\
& \mathbf{r}^{B_{k}}=\mathbf{r}^{P_{k}}-\frac{L_{k}}{2} \mathbf{a}_{k}, \quad(k=i, j)
\end{aligned}
$$

As it was previously mentioned, the contact scenarios can be mainly divided into aligned and misaligned types. The joint is considered to be aligned if the two unit vector remain parallel, therefore, the following condition must be fulfilled

$$
\tilde{\mathbf{a}}_{i} \mathbf{a}_{j}=\mathbf{0}
$$


where $\tilde{\mathbf{a}}$ denotes the skew-symmetric matrix associated with the vector $\mathbf{a}$. Taking into account the aligned case, and a configuration where axial and radial contact occur, superficial and linear contact zones are established, respectively. The contact points are denoted by $C$, with the subscripts $i$ and $j$ stand for bearing and journal, respectively, while the superscripts $a$ and $r$ denote axial and radial contact. Moreover, the superscript includes information of the extremity where it occurs. For instance, $C_{i}^{B, a}$ is the contact point of the bearing for axial contact in end $B$.

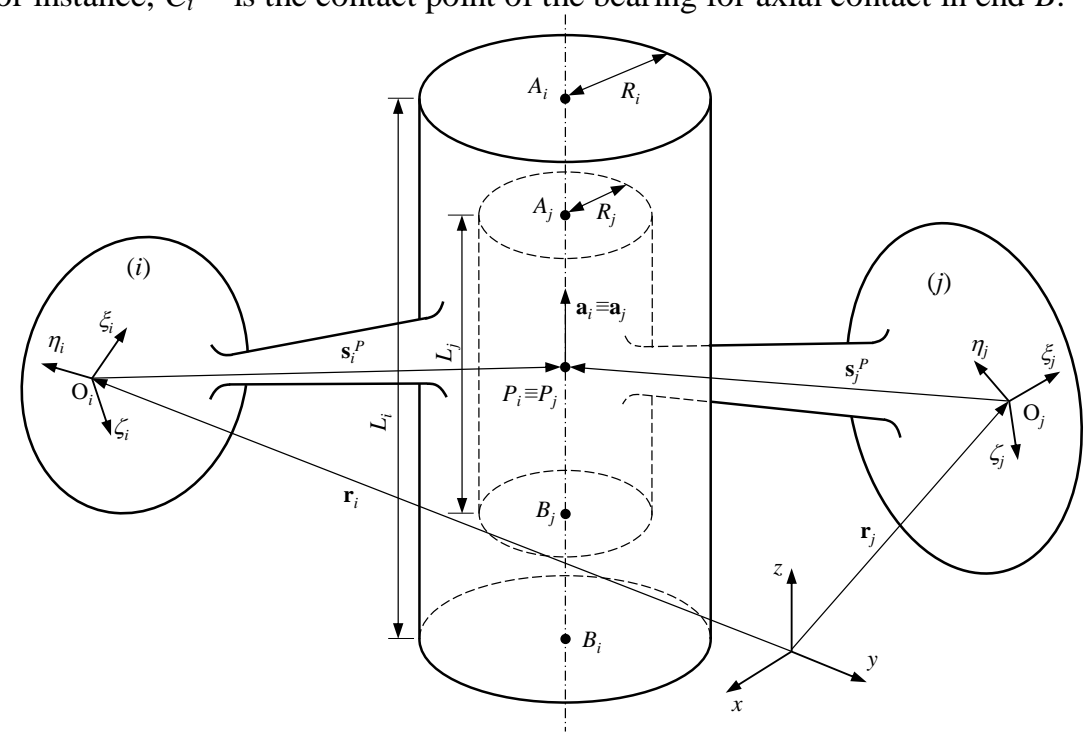

Fig. 1 General configuration of a spatial revolute joint with clearance

Regarding the radial contact, the center point of the contact line is at half of the length of the journal. Moreover, the normal unit vector can be defined as follows

$$
\mathbf{n}_{\mathrm{v}}=\frac{\left(\mathbf{r}^{P_{j}}-\mathbf{r}^{P_{i}}\right)-\left(\mathbf{r}^{P_{j}}-\mathbf{r}^{P_{i}}\right)^{T} \mathbf{a}_{i} \mathbf{a}_{i}}{\left\|\left(\mathbf{r}^{P_{j}}-\mathbf{r}^{P_{i}}\right)-\left(\mathbf{r}^{P_{j}}-\mathbf{r}^{P_{i}}\right)^{T} \mathbf{a}_{i} \mathbf{a}_{i}\right\|}
$$

Thus, the contact points can be determined by the following expressions

$$
\begin{gathered}
\mathbf{r}^{C^{r}}=\mathbf{r}^{P_{i}}+\left(\mathbf{r}^{P_{j}}-\mathbf{r}^{P_{i}}\right)^{T} \mathbf{a}_{i} \mathbf{a}_{i}+R_{i} \mathbf{n}_{\mathrm{v}} \\
\mathbf{r}^{C_{j}^{r}}=\mathbf{r}^{P_{j}}+R_{j} \mathbf{n}_{\mathrm{v}}
\end{gathered}
$$

In what concerns to the axial contact, the contact area is a circle and the center point coincides with the center of the base of the journal. Therefore, considering a contact in the end $B$, the coordinates of the contact points are given by

$$
\mathbf{r}^{C_{i}^{B, a}}=\mathbf{r}^{B_{j}}+\left(\mathbf{r}^{B_{i}}-\mathbf{r}^{B_{j}}\right)^{T} \mathbf{a}_{i} \mathbf{a}_{i}
$$




$$
\mathbf{r}^{C_{j}^{B, a}}=\mathbf{r}^{B_{j}}
$$

When the alignment of the joint does not exist, the contact detection is a more complex task. Each extremity of the journal can collide with the lateral and top walls of the bearing. A misaligned joint with axial and radial contact is represented in Fig. 2, and it is here considered as an example to apply the methodology.

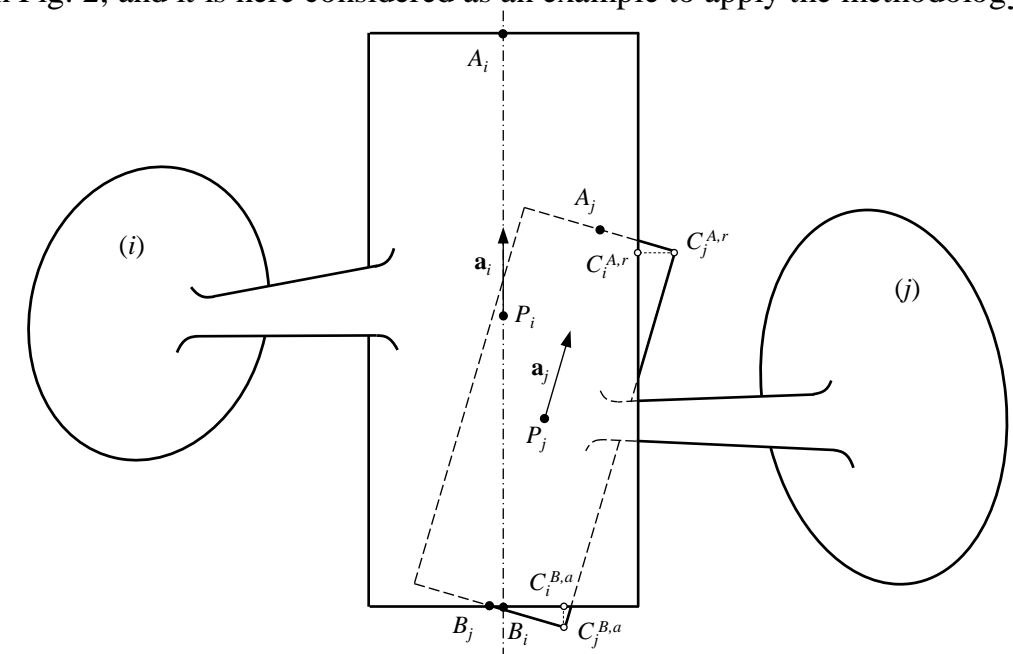

Fig. 2 Configuration of a spatial revolute joint misaligned with axial and radial contact

Regarding the axial contact, it can be defined as the intersection of a circumference (base of the journal) and a plane (base of the bearing), analytically given by

$$
\begin{gathered}
\left\{\begin{array}{c}
\left\|\mathbf{x}-\mathbf{r}^{B_{j}}\right\|^{2}=R_{j}{ }^{2} \\
\mathbf{a}_{j}{ }^{T} \mathbf{x}=\mathbf{a}_{j}{ }^{T} \mathbf{r}^{B_{j}}
\end{array}\right. \\
\mathbf{a}_{i}{ }^{T} \mathbf{x}=\mathbf{a}_{i}{ }^{T} \mathbf{r}^{B_{i}}
\end{gathered}
$$

where $\mathbf{x}$ represents the coordinates of a point belonging to each element. Both Eq. (10) and Eq. (11) can be solved together in order to find the intersection points analytically. The solution can hold the empty set, one or two solutions, respectively, no contact, tangent contact or penetration. If the solution is given by two points, $I_{1}$ and $I_{2}$, their middle point and the center of the base of the journal form a vector that defines the direction of the contact point, as

$$
\mathbf{n}_{\mathrm{d}}=\frac{\frac{1}{2}\left(\mathbf{r}^{I_{1}}+\mathbf{r}^{I_{2}}\right)-\mathbf{r}^{B_{j}}}{\left\|\frac{1}{2}\left(\mathbf{r}^{I_{1}}+\mathbf{r}^{I_{2}}\right)-\mathbf{r}^{B_{j}}\right\|}
$$

Thus, the contact point in the journal can be given as 


$$
\mathbf{r}^{C_{j}^{B, a}}=\mathbf{r}^{B_{j}} \pm R_{j} \mathbf{n}_{\mathrm{d}}
$$

where the correct sign returns a point outside the bearing. Hence, the contact point in the bearing is calculated with the following expression

$$
\mathbf{r}^{C_{i}^{B, a}}=\mathbf{r}^{C_{j}^{B, a}}+\left(\mathbf{r}^{B_{i}}-\mathbf{r}^{C_{j}^{B, a}}\right)^{T} \mathbf{a}_{i} \mathbf{a}_{i}
$$

In the case of radial contact, the problem can be converted into the intersection of a circumference (base of the journal) and a cylindrical surface (lateral wall of the bearing) which can be defined, respectively, as

$$
\begin{gathered}
\left\{\begin{array}{l}
\left\|\mathbf{x}-\mathbf{r}^{A_{j}}\right\|^{2}=R_{j}{ }^{2} \\
\mathbf{a}_{j}{ }^{T} \mathbf{x}=\mathbf{a}_{j}{ }^{T} \mathbf{r}^{A_{j}}
\end{array}\right. \\
\left\|\tilde{\mathbf{a}}_{i}\left(\mathbf{x}-\mathbf{r}^{P_{i}}\right)\right\|^{2}=R_{i}{ }^{2}
\end{gathered}
$$

Contrary to the previous case, this nonlinear system cannot be solved analytically, therefore, the intersection points are obtained by an iterative procedure. The number of solutions can vary from zero to four, although, considering a small angle between the bearing and the journal, the maximum number of solutions reduces to two. Similarly to the axial contact detection, when penetration occurs, an auxiliary vector is calculated using an expression identical to Eq. (12). Thus, the contact point in the journal can be obtained, in an analogous manner, as

$$
\mathbf{r}^{C_{j}^{A, r}}=\mathbf{r}^{A_{j}} \pm R_{j} \mathbf{n}_{\mathrm{d}}
$$

The normal unit vector to the contact can be expressed as

$$
\mathbf{n}_{\mathbf{v}}=\frac{\left(\mathbf{r}^{\mathrm{C}_{j}^{A, r}}-\mathbf{r}^{P_{i}}\right)-\left(\mathbf{r}^{\mathrm{C}_{j}^{A, r}}-\mathbf{r}^{P_{i}}\right)^{T} \mathbf{a}_{i} \mathbf{a}_{i}}{\left\|\left(\mathbf{r}^{\mathrm{C}_{j}^{A, r}}-\mathbf{r}^{P_{i}}\right)-\left(\mathbf{r}^{\mathrm{C}_{j}^{A, r}}-\mathbf{r}^{P_{i}}\right)^{T} \mathbf{a}_{i} \mathbf{a}_{i}\right\|}
$$

Hence, the contact point in the bearing can be given as follows

$$
\mathbf{r}^{C_{i}^{A, r}}=\mathbf{r}^{P_{i}}+\left(\mathbf{r}^{C_{j}^{A, r}}-\mathbf{r}^{P_{i}}\right)^{T} \mathbf{a}_{i} \mathbf{a}_{i}+R_{i} \mathbf{n}_{\mathrm{v}}
$$

It should be noted that the contact points were determined according to the example of Fig. 2. For the remaining contacts, analogous expressions must be employed. At this point, the contact kinematics for each collision can be characterized through the contact points in the bearing and journal, respectively, $C_{i}$ and $C_{j}$.

The normal unit vector of the contact was already defined for some cases, but it can be, generically, given as

$$
\mathbf{n}_{\mathrm{v}}=\left(\mathbf{r}^{C_{j}}-\mathbf{r}^{C_{i}}\right) /\left\|\left(\mathbf{r}^{C_{j}}-\mathbf{r}^{C_{i}}\right)\right\|
$$


The penetration depth, $\delta$, can be expressed by

$$
\delta=\left\|\left(\mathbf{r}^{C_{j}}-\mathbf{r}^{C_{i}}\right)\right\|
$$

Moreover, the penetration velocity, $\dot{\delta}$, can be determined as follows

$$
\dot{\delta}=\left(\dot{\mathbf{r}}^{C_{j}}-\dot{\mathbf{r}}^{C_{i}}\right)^{T} \mathbf{n}_{\mathrm{v}}
$$

where $\dot{\mathbf{r}}^{C_{i}}$ and $\dot{\mathbf{r}}^{C_{j}}$ are the linear velocities of the bearing and journal in the contact points. Finally, the relative tangential velocity can be obtained recurring to the following expression

$$
\mathbf{v}_{\mathrm{T}}=\left(\dot{\mathbf{r}}^{C_{j}}-\dot{\mathbf{r}}^{C_{i}}\right)-\left(\dot{\mathbf{r}}^{C_{j}}-\dot{\mathbf{r}}^{C_{i}}\right)^{T} \mathbf{n}_{\mathrm{v}} \mathbf{n}_{\mathrm{v}}
$$

The intra-joint contact forces developed in the revolute joint with clearance is calculated using adequate normal and tangential contact models, respectively, an elastic Hertzian model [5, 6] and a Coulomb-based approach [7].

\section{Numerical Example}

In order to validate this methodology, a spatial slider-crank mechanism is considered as an example of application, as depicted in Fig. 3a. The geometric and inertial properties of each body are listed in Table 1. The initial conditions of the mechanism consider the crank in a vertical position and moving with an angular velocity of $2 \pi \mathrm{rad} / \mathrm{s}$. Since it has only one degree of freedom, the remaining coordinates and velocities are determined such the kinematic constraints of an ideal mechanism are fulfilled. During the simulation, the system is only under the actuation of gravitational and inertial forces, with no input torque applied.

In what concerns to the revolute joint with clearance, it is located at the connection between the crank and the ground. The bearing is placed in the crank, while

\begin{tabular}{|c|c|c|c|c|c|}
\hline \multirow{2}{*}{ Body } & \multirow{2}{*}{ Length $[\mathrm{m}]$} & \multirow{2}{*}{ Mass $[\mathrm{kg}]$} & \multicolumn{3}{|c|}{ Principal Moments of Inertia $\left[\mathrm{kg} \mathrm{m}^{2}\right]$} \\
\hline & & & $I_{\xi \xi}$ & $I_{\eta \eta}$ & $I_{\zeta \zeta}$ \\
\hline Crank & 0.10 & 0.12 & 0.0001 & 0.0001 & 0.00001 \\
\hline Rod & 0.29 & 0.5 & 0.004 & 0.0004 & 0.004 \\
\hline Slider & - & 0.5 & 0.0001 & 0.0001 & 0.0001 \\
\hline
\end{tabular}
the journal belongs to the ground. The joint's properties are displayed in Table 2.

Table 1. Dimensional and inertia properties of each body

The impact of modeling a clearance joint is examined through the motion of the slider, since it represents the kinematic output of this mechanical system. Therefore, the position, velocity and acceleration of the slider are displayed in Fig. 3b-d, and compared to the ideal case. The impacts of the clearance joint highly affects the motion of the mechanism, as reported in Fig. $3 \mathrm{~d}$ with the peaks of acceleration. 


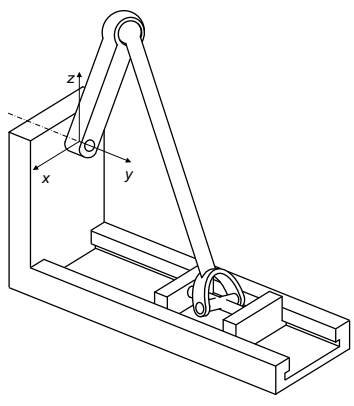

(a)

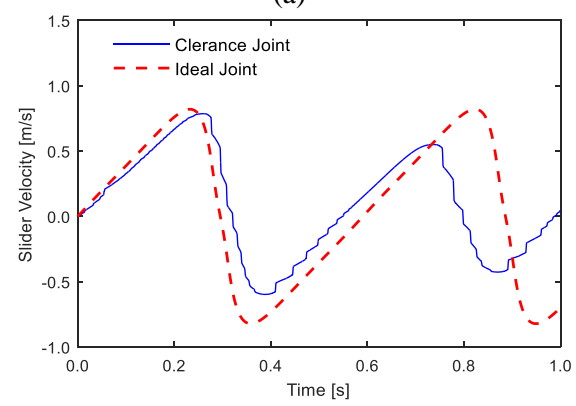

(c)

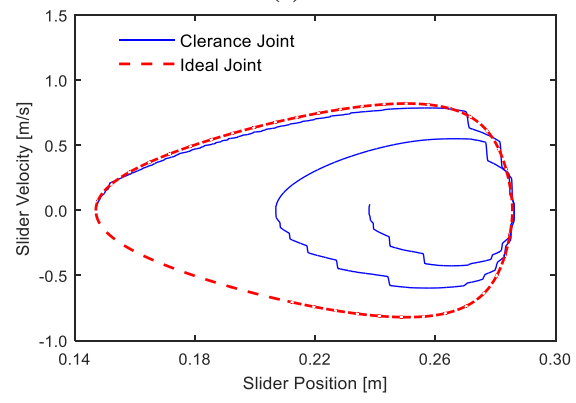

(e)

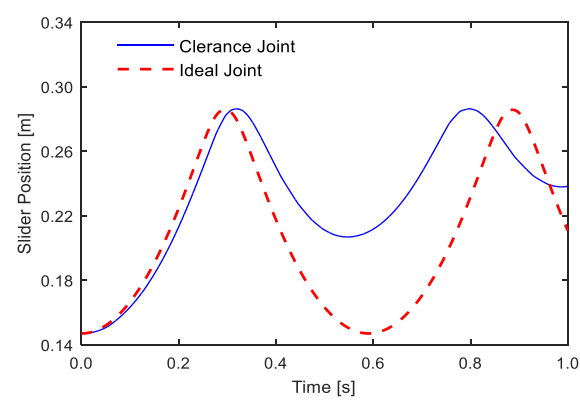

(b)

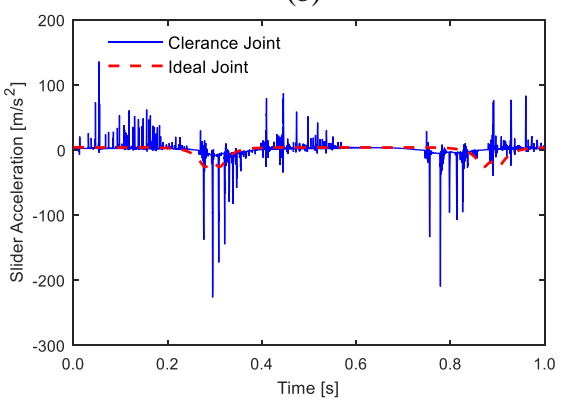

(d)

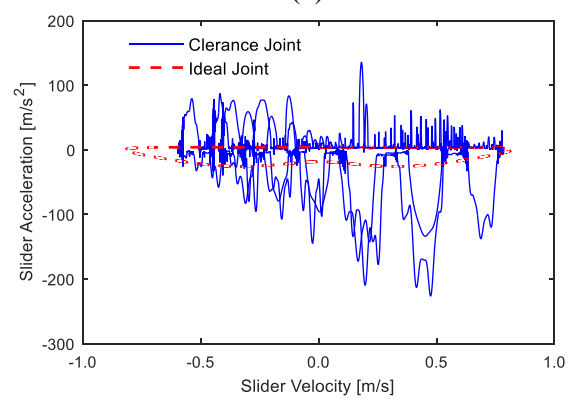

(f)

Fig. 3 (a) Representation of slider-crank mechanism. Comparison of the motion of the slider: (b) position, (c) velocity, (d) acceleration, and phase portraits of (e) pos-vel and (f) vel-acc

Table 2. Parameters for simulation with the revolute clearance joint

\begin{tabular}{lclc}
\hline Parameter & Value & Parameter & Value \\
\hline Bearing Radius, $R_{i}$ & $10.0 \times 10^{-3} \mathrm{~m}$ & Bearing Length, $L_{i}$ & $20.0 \times 10^{-3} \mathrm{~m}$ \\
\hline Journal Radius, $R_{j}$ & $9.5 \times 10^{-3} \mathrm{~m}$ & Journal Length, $L_{j}$ & $19.9 \times 10^{-3} \mathrm{~m}$ \\
\hline Radial Clearance, $c_{r}$ & $5 \times 10^{-4} \mathrm{~m}$ & Axial Clearance, $c_{a}$ & $5 \times 10^{-5} \mathrm{~m}$ \\
\hline Contact Stiffness, $K$ & $6.71 \times 10^{10} \mathrm{~N} / \mathrm{m}^{3 / 2}$ & Integration Algorithm & ode 45 \\
\hline Coefficient of Restitution, $c_{\mathrm{e}}$ & 0.9 & Reporting Time Step & $1 \times 10^{-5} \mathrm{~s}$ \\
\hline Coefficient of Friction, $\mu$ & 0.1 & Simulation Time & $1 \mathrm{~s}$ \\
\hline
\end{tabular}


The contact-impact phenomenon also produces energy losses, which results in the reduction of the amplitude of the slider's position and in the decrease of velocity, as depicted in Fig. 3b-c. The phase portraits of position-velocity and velocityacceleration presented in Fig. 3e-f demonstrate that the system's motion is nonlinear. This aspect is more evident in the latter due to the dense overlapping lines.

\section{Concluding Remarks}

A methodology for modeling spatial revolute joints with axial and radial clearance has been described in this work. The different contact scenarios were identified and the procedure for the evaluation of the contact points was defined. This formulation mainly consists on the geometric definition of the contacting bodies through analytical expressions, and on their utilization to evaluate the contact kinematics. A spatial slider-crank mechanism was employed to validate this formulation, and the results were compared to an ideal joint scenario.

Acknowledgments The first author thanks to the Portuguese Foundation for Science and Technology through the PhD grant (PD/BD/114154/2016). This work has been supported by the Portuguese Foundation for Science and Technology with the reference project UID/EEA/04436/2013, by FEDER funds through the COMPETE 2020 - Programa Operacional Competitividade e Internacionalização (POCI) with the reference project POCI-01-0145-FEDER-006941.

\section{References}

1. Flores, P., Ambrósio, J., Pimenta Claro, J.C. and Lankarani, H.M.: Kinematics and Dynamics of Multibody Systems with Imperfect Joints. In: Lecture Notes in Applied and Computational Mechanics, Springer Verlag (2008)

2. Ma, J., Qian, L., Chen, G. and Li, M.: Dynamic analysis of mechanical systems with planar revolute joints with clearance. Mechanism and Machine Theory, 94, 148-164 (2015)

3. Tian, Q., Xiao, Q., Sun, Y., Hu, H., Liu, H. and Flores, P.: Coupling dynamics of a geared multibody system supported by ElastoHydroDynamic lubricated cylindrical joints. Multibody System Dynamics, 33(3), 259-284 (2015)

4. Marques, F.: Frictional Contacts in Multibody Dynamics. MSc Dissertation. University of Minho, Portugal (2015)

5. Lankarani, H.M. and Nikravesh, P.E.: A contact force model with hysteresis damping for impact analysis of multibody systems. Journal of Mechanical Design, 112(3), 369-376 (1990)

6. Alves, J., Peixinho, N., Silva, M.T., Flores, P. and Lankarani, H.M.: A comparative study of the viscoelastic constitutive models for frictionless contact interfaces in solids. Mechanism and Machine Theory, 85, 172-188 (2015)

7. Marques, F., Flores, P. and Lankarani, H.M.: On the Frictional Contacts in Multibody System Dynamics. In: Multibody Dynamics, Computational Methods in Applied Sciences 42, 67-91 (2016) 